\title{
AMBIENTES VIRTUAIS DE APRENDIZAGEM COMO FERRAMENTAS DE APOIO EM CURSOS PRESENCIAIS E A DISTÂNCIA
}

Lélis Maia de Brito, Universidade Federal de Ouro Preto, lelis@cead.ufop.br José Renato Giuberti Júnior, Instituto Federal do Espírito Santo, rgiuberti@gmail.com Silvane Guimarães Silva Gomes, Universidade Federal de Viçosa, silvane@ufv.br João Batista Mota, Universidade Federal de Viçosa, joaomota@ufv.br

Resumo: As tecnologias vêm sendo incorporadas na educação presencial e a distância com o objetivo de enriquecer o processo de ensino-aprendizagem. Uma dessas inovações compreende-se nos ambientes virtuais de aprendizagem (AVAs), que são espaços virtuais nos quais os usuários, os educadores e os educandos podem interagir por meio de diversas ferramentas. A ideia é a de que esses ambientes criem novas possibilidades de aprendizagem ao aluno em face às mudanças tecnológicas que vêm ocorrendo na sociedade. Dessa forma, o objetivo deste trabalho foi discutir sobre a importância dessas tecnologias no processo de ensino-aprendizagem e apresentar um caso específico de ambiente virtual, o PVANet, da Universidade Federal de Viçosa. A utilização desse ambiente tem sido ampla em cursos presenciais e a distância atendendo às perspectivas de usabilidade e interatividade dispondo das principais ferramentas de interação entre os usuários. Foi possível apontar situações no processo de ensino aprendizagem que atende às perspectivas de usabilidade e interatividade possibilitando interação entre professor e estudante. Assim como, percebeu-se que a inserção de novas interfaces no AVA possibilitaria a reorganização da metodologia de ensino a partir da seleção dos meios mais apropriados para determinada situação de ensino-aprendizagem.

Palavras-chave: Educação. Tecnologia. Ambiente Virtual de Aprendizagem (AVA).

\section{VIRTUAL LEARNING ENVIRONMENTS AS SUPPORT TOOLS IN CLASSROOM AND DISTANCE COURSES}

\begin{abstract}
The technologies are being used in education and distance learning with the objective to enrich the teaching-learning process. One such innovation is understood in virtual learning environments (VLEs), which are virtual spaces where users, educators and students can interact through various tools. The idea is that these virtual learning environments create new learning opportunities to students according to the technological changes which are changing the society. Thus, the aim of this study was discuss the importance of these technologies in the teaching-learning process and present a specific case of the virtual learning environment, the PVANet, by Federal University of Viçosa. The use of this virtual environment has been wide in distance and classroom courses answer the prospects of usability and interactivity using the main interaction tools between the users. It was possible identify situations in the learning process which meets the perspectives of usability and interactivity enabling interaction between teacher and student. Thus, was observed that the insertion of new interfaces in AVA enable reorganizing the teaching methodology from the selection of the most appropriate for a given situation of teaching and learning.
\end{abstract}

Keywords: Education. Technology. Learning Management Systems (LMS). 


\section{INTRODUÇÃO}

A educação tem apresentado avanços em seu processo de ensino-aprendizagem, o que fica evidente pela utilização cada vez mais intensa das tecnologias da informação e comunicação (TICs). Essas ferramentas têm sido incorporadas às atividades educacionais, com o objetivo de criar novas possibilidades de ensino ao professor e melhorar a aprendizagem do aluno. Além disso, a informática pode ser utilizada como uma ferramenta que desperta o interesse em aprender no aluno, possibilitando novas condições de aprendizagem.

$\mathrm{O}$ resultado das pesquisas sobre apoio das tecnologias à aprendizagem colaborativa (CSCL) foi a criação de um espaço virtual de interação entre professor e aluno: os ambientes virtuais de aprendizagem (AVAs). Nesses ambientes, são incorporadas ferramentas da web, como o correio eletrônico, os fóruns, as salas de discussão ou chats e outras ferramentas de envio de arquivos e avaliação das atividades e participações dos integrantes, por meio de relatórios e gerenciamentos.

Os AVAs podem dispor de todas as classificações de softwares educativos. Isto é, no mesmo ambiente é possível criar e disponibilizar ferramentas instrucionistas (são utilizadas visando à apresentação de informações aos alunos ou estabelecendo algum tipo de instrução) e construcionistas (permitem ao aluno criar ou simular ações dentro do ambiente), as quais têm por objetivo central valorizar o processo de ensinoaprendizagem. Nessa mesma temática, Cristovão e Nobre (2011), apresentam cinco classificações de softwares educativos em suas ferramentas de ensino: a apresentação, a consulta, as perguntas e respostas, a comunicação e a cooperação.

Os ambientes educacionais têm sido utilizados como espaços para desenvolver novas práticas de ensino, tanto em cursos presenciais quanto em cursos a distância. Dessa forma, diversos autores complementam que é preciso dar condições à instituição de ensino incorporar essas novas tecnologias para que possa modificar seus padrões educacionais. Caso contrário, ela poderá ser considerada como excluída desse inovador processo de ensino-aprendizagem e da relação entre professor e aluno.

Em face da agenda de discussão sobre a utilização de ambientes virtuais em cursos presenciais e a distância, este estudo discute a importância dos AVAs no processo de ensino-aprendizagem. Além disso, descreve, como objeto de estudo, um ambiente virtual de aprendizagem, elaborado e de uso exclusivo da Universidade Federal de Viçosa, intitulado PVANet, utilizado em cursos presenciais e a distância na instituição.

\section{AMBIENTES VIRTUAIS DE APRENDIZAGEM (AVA)}

A educação tem apresentado avanços no que diz respeito ao uso de Tecnologias de Informação e Comunicação (TIC). A utilização dessas tecnologias educacionais visa inovar as formas de ensino e enriquecer o aprendizado "tornando o processo de ensinar e aprender mais agradável, ágil e útil para o mundo atual” (Lovatte e Nobre, 2011). Nesse sentido, esse conjunto de ferramentas tecnológicas vem sendo utilizado para dinamizar o processo de ensino-aprendizagem nos cursos a distância e também presenciais. O que para Franco (2003) e Moore (1993) significa que essa concepção de distância educacional não está ligada apenas ao fator físico, geográfico, mas na visão da comunicação, interação e participação. Nessa ideia, Moran (2002) destaca que o advento das tecnologias de comunicação virtual vem alterando o conceito de presencialidade na educação.

Segundo Pereira et al. (2011) a utilização de ambientes virtuais de aprendizagem, também conhecidos por "Sistemas de Gerenciamento de Cursos 
(Learning Management System)” Ferreira (2009), vem se apresentando como uma nova ferramenta tecnológica que atende às inovações na educação. Para complementar, Dillenburg (2011) destaca que os ambientes virtuais de aprendizagem são "softwares desenvolvidos para o gerenciamento da aprendizagem via web a partir da Comunicação Mediada por Computador (CMC)".

Os ambientes virtuais de aprendizagem, segundo alguns autores (Sarmento et al. (2011), Barbosa (2005) e Castro Filho et al. (2005)) compreendem-se na integração de um conjunto de tecnologias digitais que possibilita a construção de um ambiente ou software educativo no qual é possível promover a informação em conhecimento aos seus integrantes de forma individual ou coletiva. Dessa forma, AVA pode ser, segundo Dillenburg e Teixeira (2011), reestruturado como uma "sala de aula presencial física para o meio on-line" usando "tecnologias adequadas para propiciar aos aprendizes novas ferramentas que facilitem a aprendizagem".

Para Franco et al. (2003) e Sarmento et al. (2011), os ambientes incorporam ferramentas da web como o correio eletrônico, os fóruns, as salas de discussão ou também denominadas de chat e outras ferramentas de envio de arquivos e avaliação das atividades e participações dos integrantes por meio de relatórios e gerenciamentos. Pequeno et al. (2004) avança nas discussões ressaltando que "o uso pedagógico dessas tecnologias pode ser notado através do crescimento dos ambientes de texto colaborativos, wiki, e das experiências de produção multimídia, como, por exemplo, em blogs e no site youtube”. No entanto, Sarmento et al. (2011) relatam que o mais importante em um ambiente virtual de aprendizagem é a facilidade do uso das ferramentas tecnológicas. Ou seja, o ambiente precisa desenvolver padrões de usabilidade, que segundo o autor, compreende-se no grau de facilidade em que o sistema possibilita a interação do usuário com as ferramentas e as interações com os demais usuários.

De maneira geral o que se tem discutido sobre a usabilidade de ambientes virtuais compreende-se nas estruturas, processos e resultados. Essas caracterizações, segundo Laguardia (2007) referem-se às "condições em que a aprendizagem se realiza, nos modos pelos quais os estudantes são capazes de interagir e o alcance dos objetivos e metas propostas”. Complementando essa ideia de usabilidade, Filho et al. (2008) destaca a norma técnica da Associação Brasileira de Normas Técnicas (ABNT) ISO 9241 que estabelece um padrão de requisitos que o sistema interativo apresente um contexto de operação para que as atividades executadas no ambiente possam ser eficazes, eficientes e agradáveis. Dentre os requisitos exigidos destacam-se, segundo o autor, a adaptabilidade, a condução, o controle e a homogeneidade/coerência das ferramentas apresentadas pelo ambiente virtual.

Nesse sentido, o que se discute é a importância do papel das tecnologias da informação e comunicação associadas aos ambientes virtuais de aprendizagem no processo de ensino-aprendizagem. É preciso que as ferramentas tecnológicas possam oferecer um conjunto de funcionalidades que auxiliam o educador e o educando a promover as interações por meio desse ambiente. Além dessa interação tem-se discutido de que maneira os processos de ensino-aprendizagem ocorrem nesses ambientes e quais são os tipos de aprendizagem e os canais utilizados em ambientes de aprendizagem que facilitem o ensino e a aprendizagem dos usuários.

\section{ENSINO-APRENDIZAGEM EM AVA}

No AVA há a utilização de novas práticas pedagógicas visando minimizar as limitações de tempo e espaço entre os atores educacionais, professor e aluno. Dentre as 
ações que visam discutir e aprimorar o papel da tecnologia no ambiente educacional destaca-se a Aprendizagem Colaborativa Mediada por Computador (Computer Supported Collaborative Learning - CSCL) que tem por objetivo criar novas ferramentas que auxiliam no desenvolvimento do ensino-aprendizagem.

Para Cristovão e Nobre (2011), "a verdadeira função do aparato educacional não é a de ensinar, mas sim, a de criar condições de aprendizagem”. Dessa forma, Motta e Gava (2011) apontam que esse ambiente educativo "contribui sobremaneira para o desenvolvimento dos novos processos cognitivos".

Os estilos de aprendizagem podem ser considerados como maneiras de se obter conhecimentos. Belhot (2005) define os estilos de aprendizagem como um processo, no qual o aluno, de forma distinta e habitual, associa diversas informações, "habilidades e atitudes através do estudo ou da experiência”. Assim, em função dessa diferenciação, entre alunos, é preciso compreender de que forma e por qual meio ou ferramentas o processo de ensino-aprendizagem valorizará essa forma de desenvolvimento do conhecimento.

Reis (2011) a partir dos estudos de Paulo Freire, Skinner, Rogers, Piaget, Bruer e Vygotsky definem algumas abordagens da aprendizagem, tais como a abordagem tradicional, a comportamentalista, a humanista, a cognitivista e a sociocultural.

Para esses autores, a abordagem tradicional "é caracterizada pela concepção da educação como um produto, a partir de ideias selecionadas e organizadas, logicamente”. $\mathrm{Na}$ abordagem comportamentalista (Skinner), o "ensinar consiste num arranjo de contingência de reforço sob as quais os estudantes aprendem e é de responsabilidade do professor assegurar a aquisição do comportamento”. Além disso, nessa concepção, “o professor tem a responsabilidade de planejar e desenvolver o sistema de ensinoaprendizagem de forma que o desempenho do aluno seja maximizado, levando em consideração fatores como economia de tempo, esforços e custos”. Para a abordagem humanista (Rogers), “o professor, em si, transmite o conteúdo e dá a assistência, ou seja, facilita a aprendizagem. Nesse tipo, o aluno é o responsável pelas formas de controle da sua aprendizagem a qual é desenvolvida a partir das suas próprias experiências”. Na abordagem cognitivista (Piaget e Bruer), a "ênfase se dá por meio de processos cognitivos e na investigação científica, separada dos problemas sociais contemporâneos e predominantemente interacionista”. Complementando, Reis (2011) cita os estudos de Vygostky, os quais explicam que "a formação se dá numa relação dialética entre o sujeito e a sociedade a seu redor no sentido de que o homem modifica o ambiente e o ambiente modifica o homem, ou seja, há a experiência pessoalmente significativa nesse processo de aprendizagem”. Por fim, a abordagem sociocultural (Paulo Freire), explica que "o homem cria a cultura na medida em que, integrando-se nas condições do seu contexto de vida, reflete sobre ela e dá respostas aos desafios que encontra, ou seja, o homem é o sujeito da educação”.

Portanto, o que se discute é que o acesso e a utilização das ferramentas tecnológicas, por meio de ambientes virtuais de aprendizagem, permitem que alunos e professores desenvolvam um processo de ensino-aprendizagem capaz de abranger qualquer dimensão de aprendizagem do aluno, seja no modelo a distância ou presencial. Assim, segundo Reis (2011), “uma ferramenta tecnológica se torna não somente um mediador passivo, pois é também um instrumento para um diálogo ativo entre o indivíduo e seu ambiente”. Dessa forma, insere-se nessa discussão a utilização de ferramentas tecnológicas que permitem a interação e a construção do conhecimento entre os alunos e os professores.

Nesse sentido, Cristovão e Nobre (2011:127) esclarecem que há basicamente duas vertentes sobre a utilização desses tipos de tecnologias na educação. A primeira 
delas compreende-se em utilizações instrucionistas, que segundo os autores, "são aquelas que privilegiam a apresentação das informações para o aluno ou uma interação do tipo instrução, na qual o aluno responde às questões propostas, tendo ou não a sua resposta qualificada". A segunda, utilização construcionista, permite a "abertura para o aluno experimentar, simular, brincar, construir a partir de um ambiente compatível com tais ações e num contexto preparado para tal”.

Levando em consideração a classificação da ferramenta tecnológica e o ambiente virtual ou software educativo há algumas classificações. As principais classificações são apresentadas no Quadro 1.

Quadro 1. Principais classificações de softwares educativos.

\begin{tabular}{|c|c|}
\hline CLASSIFICAÇÃO & DESCRIÇÃO \\
\hline Apresentação & $\begin{array}{l}\text { Exibe informações previamente estabelecidas ou então escolhidas pelo apresentador e limita, ao } \\
\text { usuário, apenas visualizar o conteúdo. }\end{array}$ \\
\hline Consulta & $\begin{array}{l}\text { Permite a consulta das informações por meio de buscas diretas por palavras chaves, na qual o } \\
\text { aprendiz interage, diretamente, com o software mantendo o controle do que é apresentado. }\end{array}$ \\
\hline Pergunta/Resposta & Permite ao usuário analisar as perguntas sucessivas das respostas sobre determinado tema. \\
\hline Tutor inteligente & $\begin{array}{l}\text { Permite identificar os passos do aprendiz construindo um modelo personalizado, a partir do } \\
\text { histórico de acesso do usuário. }\end{array}$ \\
\hline Construção & $\begin{array}{l}\text { Permite a construção de algo pela exploração autodirigida, ou mesmo sugerida pelo mediador, quer } \\
\text { seja o professor ou o próprio software educativo. }\end{array}$ \\
\hline Jogo & $\begin{array}{l}\text { Disponibiliza aspectos lúdicos que fazem o aluno envolver-se de tal forma a aumentar muito a sua } \\
\text { motivação. }\end{array}$ \\
\hline Simulação & Permite criar, ficticiamente, a realidade ou o imaginário. \\
\hline Micromundo & $\begin{array}{l}\text { Permite ao aprendiz construir a partir de um pequeno conjunto de primitivas, uma representação } \\
\text { mais completa do domínio. }\end{array}$ \\
\hline Programação & $\begin{array}{l}\text { Permite construir programas representados por comandos escritos, ou na forma visual, que podem } \\
\text { ser modificados, testados, pelo aprendiz quantas vezes forem necessárias. }\end{array}$ \\
\hline Comunicação & Ambientes que permitem e facilitam a interação entre seres humanos ou entre humanos e robôs. \\
\hline Cooperação & Permitem a construção cooperativa, de, por exemplo, um texto ou uma apresentação. \\
\hline
\end{tabular}

Fonte: Adaptado de Cristovão e Nobre (2011)

É importante destacar que os ambientes virtuais de aprendizagem podem apresentar algumas ou todas as classificações de software educativo, de acordo com os tipos ou utilizações das ferramentas tecnológicas do mesmo. De maneira geral, os ambientes virtuais dispõem de ferramentas instrucionistas e construcionistas e são classificados de acordo com suas funcionalidades, basicamente, em softwares educativos do tipo perguntas e respostas, comunicação e cooperação (ex: Moodle). 


\section{AMBIENTE DE ENSINO: PVANET}

O ambiente virtual de aprendizagem, nomeado pela equipe idealizadora, como PVANet, visa promover o processo de ensino-aprendizagem cooperativo e colaborativo mediado por computador nas disciplinas de graduação e pós-graduação nas modalidades presenciais e a distância da Universidade Federal de Viçosa-MG.

Seu nome foi originado da fusão das palavras pavilhão de aulas (PVA) e rede de internet (Net). Sua denominação, portanto, remete à ideia de extensão das salas de aulas presenciais existentes na UFV, para o ambiente virtual de aprendizagem.

O PVANet foi desenvolvido a partir de uma pesquisa em nível de mestrado do programa de pós-graduação em Ciência e Tecnologia de Alimentos da UFV. A pesquisa (Arquete, 2003) teve por objetivo apoiar o processo ensino-aprendizagem da disciplina Cibernética de Processos Bioquímicos do curso de Engenharia de Alimentos. Desenvolvido em linguagem PHP (Personal Home Page), conta com diversas ferramentas que o torna dinâmico, interativo e de fácil utilização.

Disponibilizado para toda a comunidade universitária da UFV em 2004, o AVA apresenta ferramentas e interface que possibilitam a inclusão de conteúdos, nos mais diferentes formatos (textos, apresentações narradas, vídeos, animações e simulações, interação aluno-professor síncrona e assíncrona, e acompanhamento do processo de aprendizado, via avaliações). As informações são exibidas aos seus usuários por meio das interfaces predefinidas de cada ferramenta.

Abaixo são apresentadas algumas imagens das páginas de acesso do ambiente.

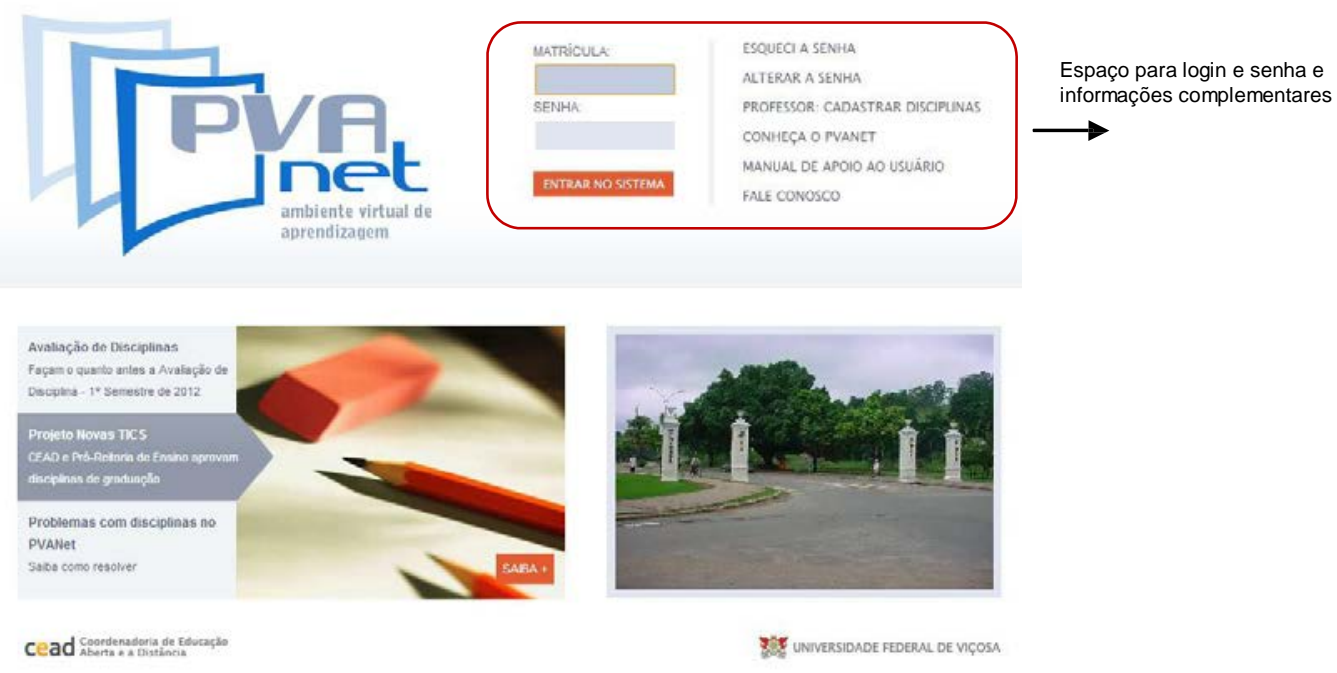

Fonte: CEAD (2012a)

Figura 1. Página de acesso ao ambiente virtual de aprendizagem PVANet

A Figura 1 apresenta a página inicial de acesso ao ambiente. Nessa página inicial o usuário poderá acessar o PVANet $^{1}$, por meio de matrícula e senha, utilizando softwares de navegação (Mozilla Firefox, Internet Explorer ou similares). Ainda na página inicial há alguns tópicos de orientação ao usuário, tais como: “esqueci a senha”, o "alterar a senha”, o "cadastro de disciplinas" pelo professor, o vídeo institucional do ambiente, o manual de apoio ao usuário e o contato direto com o suporte tecnológico do ambiente por meio do "fale conosco". Além da comunidade universitária da UFV, o AVA possibilita, também, cadastrar usuários externos ao ambiente.

\footnotetext{
${ }^{1}$ Endereço eletrônico: https://www2.cead.ufv.br/sistemas/pvanet/geral/login.php
} 
Para exemplificar as principais ferramentas do PVANet, foi utilizada a disciplina Ambientação em Educação a Distância (CDA001) do curso Técnico em Agropecuária na modalidade a distância. Ao acessar a disciplina escolhida, CDA001, a seguinte página inicial poderá ser visualizada (Figura 2).

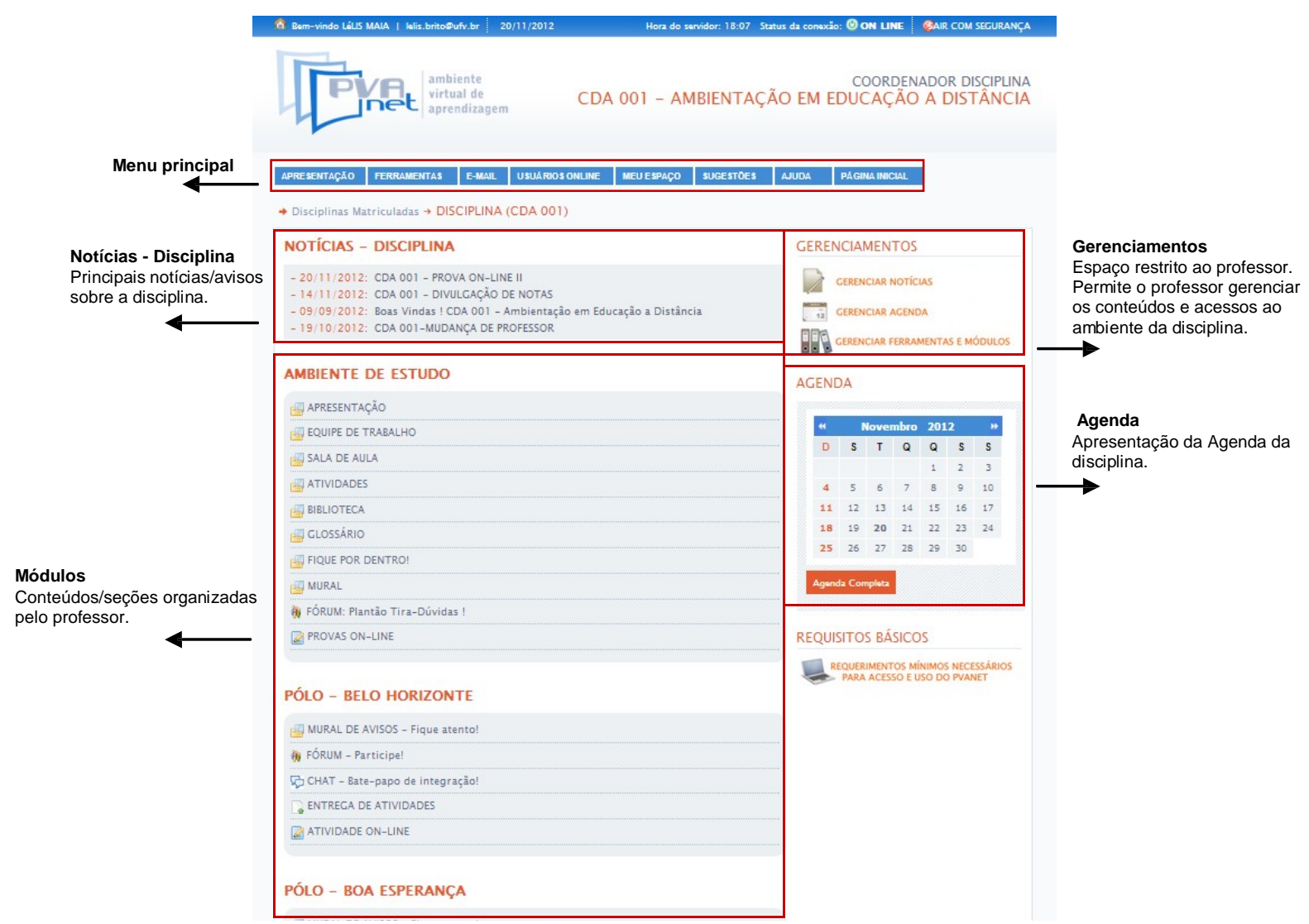

Fonte: CEAD (2012a)

Figura 2. Página inicial da disciplina Ambientação em Educação a Distância no PVANet

A Figura 2 apresenta algumas das principais ferramentas do sistema. No "menu principal”, há um conjunto de ícones de acesso rápido: é possível acessar algumas ferramentas de gerenciamento do sistema, tais como a apresentação da disciplina, as ferramentas de controle, os usuários on-line, o meu espaço, as sugestões, a ajuda, a página inicial e o sistema de $e$-mail.

No espaço "notícias", o aluno poderá visualizar os principais avisos publicados pelo professor. O espaço serve como meio de atualizar o aluno sobre os principais acontecimentos na disciplina. Logo abaixo das notícias há os "módulos": Ambiente de Estudo, Polo - Belo Horizonte e Polo - Boa Esperança. Esses módulos são gerenciados pelo professor de acordo com a organização da disciplina. Em cada módulo é possível inserir as ferramentas do ambiente conforme o planejamento pedagógico da disciplina.

Ainda de acordo com a Figura 2, em "gerenciamento", é possível inserir, editar ou excluir os módulos e as ferramentas, como por exemplo, o fórum, o chat, perguntas e respostas, envio de trabalhos, avaliação on-line e edição compartilhada de arquivo.

O fórum é uma ferramenta de comunicação assíncrona (não simultânea) que tem por objetivo promover o debate sobre determinado tema. Já o chat é um meio de comunicação síncrona por promover a comunicação simultânea entre os participantes. 
Em ambas as ferramentas, o objetivo é discutir sobre determinado tema, além de dar apoio ao aluno sobre os conteúdos estudados.

É importante destacar que o ambiente virtual de aprendizagem PVANet é utilizado como ambiente de ensino na modalidade presencial e a distância. De acordo com o seu Sistema de Gerenciamento, é utilizado por 3.267 disciplinas, sendo 155 na modalidade a distância, incluindo outros campi (Florestal e Rio Paranaíba). Ao todo são aproximadamente 16.258 estudantes, dos quais 1.258 de cursos a distância, e 1.100 professores que utilizam o ambiente de ensino. Por meio do PVANet são oferecidos cursos na modalidade a distância. Dentre eles destacam-se três cursos de aperfeiçoamento (curso básico de linux, programação em linguagem fortran e visualização científica por meio de linguagem NCL); dois cursos de licenciatura (Matemática e História); três cursos técnicos (Agropecuária, Informática e Hospedagem) e oito cursos de especialização.

\section{CONSIDERAÇÕES FINAIS}

As ferramentas disponíveis no ambiente PVANet envolvem diversas utilizações que contribuem para o processo de ensino-aprendizagem. No entanto, observa-se que essas ferramentas são utilizadas, basicamente, na visão instrucionista, ou seja, tem por objetivo apresentar um conjunto de informações ou de instruir o aluno para determinada ação. Além disso, esse ambiente, em função das ferramentas, é classificado como software educativo, predominantemente, do tipo comunicação (chat, fórum e e-mail), pergunta e resposta (pergunta/resposta, entrega de trabalhos e avaliação on-line) e cooperação (edição compartilhada de arquivo).

Nesse sentido, apresenta-se abaixo um quadro que apresenta as principais ferramentas desse ambiente e suas respectivas utilizações e classificações.

Quadro 2. Utilizações e classificações das ferramentas do ambiente PVANet

\begin{tabular}{|c|c|c|c|}
\hline \multirow{2}{*}{ FERRAMENTAS } & \multirow{2}{*}{ DESCRIÇÃO DA FERRAMENTA } & \multirow{2}{*}{ UTILIZAÇÕES } & $\begin{array}{l}\text { CLASSIFICAÇÕ } \\
\text { ES }\end{array}$ \\
\hline & & & $\begin{array}{l}\text { SOFTWARES } \\
\text { EDUCATIVOS }\end{array}$ \\
\hline Chat & $\begin{array}{l}\text { Possibilita a interação (síncrona) entre professores, } \\
\text { tutores e alunos por meio de uma sala virtual. }\end{array}$ & Instrucionista & Comunicação \\
\hline Fórum & $\begin{array}{l}\text { Possibilita a apresentação de um tema central para } \\
\text { discussão (assíncrona) entre professores, tutores e } \\
\text { alunos. }\end{array}$ & Instrucionista & Comunicação \\
\hline $\begin{array}{l}\text { Perguntas e } \\
\text { Respostas }\end{array}$ & $\begin{array}{l}\text { Possibilita a apresentação de perguntas e as respectivas } \\
\text { respostas por professores, tutores e alunos. Pode ser } \\
\text { utilizada, também, como um glossário. }\end{array}$ & Instrucionista & Pergunta/Resposta \\
\hline Sistema $e$-mail & $\begin{array}{l}\text { Possibilita a troca de mensagens (envio de arquivos e } \\
\text { gerenciamento das informações) entre professores, } \\
\text { tutores e alunos. É possível formar grupos de } e \text {-mails e } \\
\text { constatar se a mensagem foi recebida. }\end{array}$ & Instrucionista & Comunicação \\
\hline Entrega de & $\begin{array}{l}\text { Possibilita o envio de arquivos (em diversos formatos: } \\
\text { editores de texto, de apresentações ou planilhas }\end{array}$ & Instrucionista & Pergunta/Resposta \\
\hline
\end{tabular}




\begin{tabular}{cllc}
\hline trabalhos & eletrônicas, entre outros) através do ambiente. & \\
\hline $\begin{array}{c}\text { Sistema de avaliação } \\
\text { on-line }\end{array}$ & $\begin{array}{l}\text { Possibilita a realização de uma atividade (com questões } \\
\text { discursivas e objetivas) on-line. }\end{array}$ & Instrucionista & Pergunta/Resposta \\
\hline $\begin{array}{c}\text { Edição } \\
\text { compartilhada de } \\
\text { arquivo }\end{array}$ & $\begin{array}{l}\text { Possibilita compartilhar e editar textos redigidos pelos } \\
\text { usuários. }\end{array}$ & Construcionista & Cooperação \\
\hline
\end{tabular}

Fonte: Elaborado pelos autores.

As principais ferramentas utilizadas no PVANet são semelhantes àquelas disponíveis nos demais AVAs - como o Moodle. Seu uso vem promovendo a interação entre o professor e o aluno, entre a tecnologia e a informação. Porém, de acordo com as pesquisas discutidas ao longo do trabalho, é preciso que um AVA amplie suas ações, com o objetivo de promover o processo de ensino-aprendizagem por meio de ferramentas instrucionistas e também construcionistas. O que se observa é que uma das limitações do ambiente virtual em questão (PVANet) é a disponibilidade de ferramentas que possibilitam ao usuário experimentar, construir e simular ações. Embora uma ferramenta disponível no ambiente (edição compartilhada de arquivos) apresente essa classificação, ela limita-se apenas à edição de textos. Dessa forma, ressalta-se que é preciso ampliar o conjunto de ferramentas construcionistas visando dar condições de se efetivar a aprendizagem do aluno.

Apesar do conjunto de ferramentas disponíveis e da ampla utilização desse ambiente virtual no ensino presencial e a distância são incipientes as pesquisas sobre sua importância, qualidade e usabilidade. Para tanto, sugere-se como pesquisa futura compreender a facilidade e a qualidade dessas ferramentas analisando como as mesmas são utilizadas, em quais tipos de atividades e o quanto elas podem influenciar no processo de ensino-aprendizagem.

\section{REFERÊNCIAS}

ARQUETE, D.A.R. Ensino-aprendizagem de cibernética de processos bioquímicos mediado por computador. Viçosa: UFV, 2003. Dissertação de Mestrado.

BARBOSA, R.M.(org.).Ambientes Virtuais de Aprendizagem. Editora Artmed. 2005.

BELHOT, R.V. A didática no ensino de engenharia. In: Congresso Brasileiro de Ensino de engenharia, 33., 2005. Campina Grande. Anais. 2005.

CASTRO FILHO, J. A.; LOUEREIRO, R. C.; PAULA, P. S.; SARMENTO, W. W. F.; PEIXOTO, L. E.; PEQUENO, H. S. L.; ROCHA, B. T. S.;VIANA JÚNIOR, G. S. Portal Humanas: Um ambiente colaborativo para criação de projetos e comunidades virtuais para a área de Humanidades. In: Simpósio Brasileiro de Informática na Educação, 16., 2005. Juiz de Fora. Anais. 2005.

CEAD. Coordenadoria de Educação Aberta e a Distância. Universidade Federal de Viçosa. Ambiente Virtual de Aprendizagem: PVANet. Disponível em: <https://www2.cead.ufv.br/cead/scripts/professor.php.> Acesso em: nov. 2012a.

CRISTOVÃO, H.M., NOBRE, I.A. Software educativo e objetos de aprendizagem. In: NOBRE, I.A. [et al.] Informática na educação: um caminho de possibilidades e 
desafios. Instituto Federal de Educação, Ciência e Tecnologia do Espírito Santo. Serra-ES, 2011.

DILLENBURG, D.J., TEIXEIRA, A.C. Uma proposta de avaliação qualitativa em ambientes virtuais de aprendizagem. In: Simpósio Brasileiro de Informática na Educação (SBIE), 22., 2011. Aracaju. Anais. 2011.

FERREIRA, D.D., KELLER, J., STUMM, J., SANTOS, N. O uso de ferramentas estratégicas como suporte à gestão do conhecimento na educação. Novas Tecnologias na Educação. CINTED-UFRGS. v.7. n.1, julho, 2009.

FILHO, J.L., JÚNIOR KLEIN, V.J., LINS, O., COSTA, T. Ergonomia cognitiva em ambientes virtuais de aprendizagem: Uma análise da interface humano computador (IHC) do Sistema Polvo. In: Encontro Nacional de Engenharia de Produção. 28., 2008. Rio de Janeiro. Anais. 2008.

FRANCO, M.A., CORDEIRO, L.M., CASTILLO, R.A. O ambiente virtual de aprendizagem e sua incorporação na Unicamp. Educação e Pesquisa, São Paulo. v.29, n.2, p. 341-353, jul/dez. 2003.

LAGUARDIA, J., PORTELA, M.C., VASCONCELLOS, M.M. Avaliação de ambientes de aprendizagem. Revista Educação e Pesquisa. São Paulo, v.33., n.3, p. 513-530. set/dez. 2007.

LOVATTE, E.P., NOBRE, I. A importância do uso de recursos computacionais na educação do século XXI. In: NOBRE, I.A.M. [orgs]. Informática na educação: um caminho de possibilidades e desafios. Serra, ES: Instituto Federal de Educação, Ciência e Tecnologia do Espírito Santo, 2011.

MOORE, Michael G. Theory of transaction distance. In: KEEGAN, Desmond (Ed.) Theorical principles of distance education. London: Routledge, 1993.

MORAN, J.M. Pedagogia integradora do presencial-virtual. In: Congresso Internacional de Educação a Distância. 9., 2002. São Paulo. Anais. 2002.

MOTTA, G.R., GAVA, T.R. As comunidades virtuais de aprendizagem como espaço de formação docente. In: NOBRE, I.A. [et al.] Informática na educação: um caminho de possibilidades e desafios. Instituto Federal de Educação, Ciência e Tecnologia do Espírito Santo. Serra-ES, 2011.

PEQUENO, M., LOUREIRO, R.C., SILVA, C. Modelo para gestão e implementação de ambientes virtuais de aprendizagem numa perspectiva de interface adaptativa. In: Congresso de Educación a Distancia CREAD MERCOSUR/SUL. 8., 2004. Argentina. Anais. 2004.

PEREIRA, A.T., SCHMITT, V., DIAS, M.R. Ambientes Virtuais de Aprendizagem. In: Educação a Distância e Ambientes de Aprendizagem. Instituto Federal do Espírito Santo. Centro de Educação a Distância. 2011.

REIS, E. Aprendizagem e docência digital. In: NOBRE, I.A. [et al.] Informática na educação: um caminho de possibilidades e desafios. Instituto Federal de Educação, Ciência e Tecnologia do Espírito Santo. Serra-ES, 2011.

SARMENTO, W.F., HARRIMAN, C.L., RABELO, K.F., TORRES, A.B. Avaliação de usabilidade no processo de desenvolvimento contínuo em ambientes virtuais de aprendizagem: um estudo de caso com o ambiente Solar. In: Simpósio Brasileiro de Informática na Educação (SBIE). 22., 2011. Aracaju. Anais. 2011. 